\title{
Numerical Investigation of a Transverse Jet in a Supersonic Crossflow using Large Eddy Simulation
}

\author{
Benoit Fiorina* \\ Center for Turbulence Research, Stanford University, Stanford, CA 94305-4035, USA \\ Sanjiva K. Lele ${ }^{\dagger}$ \\ Department of Aeronautics and Astronautics, Stanford University, Stanford, CA 94305-4035, USA
}

We are developing LES capability for the simulation of mixing and combustion of transversely injected fuel jets in hot supersonic crossflows. This paper describes the numerical algorithm being used and its performance on several problems involving shock wave dynamics in different configurations. Also shown are results from preliminary calculations of a transitional transverse jet injected into a supersonic crossflow computed on a coarse mesh. These results are discussed and similarity with the experimental observations are noted. Further developments, such as inclusion of the jet hole geometry, simulation of a turbulent boundary layer upstream of the injector, and higher overall resolution in the jet shear-layers are planned for the future. These would enable detailed comparisons with the available experimental data. The numerical algorithm which is based on an extension of the approach proposed by Cook \& Cabot $^{1}$ for shock-turbulence interaction is found to be suitable for LES of complex supersonic flows.

\section{Nomenclature}

$c_{p} \quad$ Specific heat at constant pressure

E Total enregy

$M \quad$ Mach number

$p \quad$ Pressure

$T$ Temperature

$R \quad$ Gas constant

$s \quad$ Entropy

$\underline{S} \quad$ Strain rate tensor

$S \quad$ Magnitude of the strain rate tensor

$t \quad$ Time

$u \quad$ Velocity vector

$\rho \quad$ Density

$\lambda \quad$ Thermal conductivity

$\gamma \quad$ Ratio of specific heats

$\mu_{l} \quad$ Fluid viscosity

$\mu_{s} \quad$ Non linear artificial shear viscosity

$\mu_{b} \quad$ Non linear artificial bulk viscosity

$\chi_{\rho} \quad$ Non linear artificial diffusivity

$\underline{\tau} \quad$ Viscous stress tensor

$\bar{\delta} \quad$ Unit tensor

$\Delta \quad$ Grid spacing

*Post-doc

$\dagger$ Professor 


\section{Introduction}

Due to the limited flow residence time inside a SCRAMJET combustor, ignition and flame-holding capabilities are critical to the development of supersonic air-breathing engines. An efficient propulsion system requires an enhanced mixing of fuel and air. One of the simplest possibility for enhanced mixing is the use of transverse injection of fuel from wall orifices. Numerical simulations of this configuration are promising tools to help in the design of the combustion chamber. ${ }^{2}$ For instance Mitani and Kouchi ${ }^{3}$ recently reported RANS simulations of an hydrogen-fueled SCRAMJET engine for Mach 6 flight condition. Following a LES approach, Lavante et al. ${ }^{4}$ succeed to recover the complex flow structure of a supersonic airflow with transverse hydrogen injection. DES capabilities to simulate the mixing of jets in supersonic crossflows has been recently investigated by Perterson et al. ${ }^{5}$

Flow structures resulting from a jet's penetration into a supersonic crossflow are illustrated on Fig. 1. After leaving the injection hole, the jet expands and form a Mach disk. Due to the blockage of the free stream supersonic flow by the transverse jet, a bow shock is also produced. It causes the upstream boundary layer to separate and leads to the formation of a recirculation zone where the fuel and the air mix under subsonic conditions. Simulation of such complicated flow are very challenging from an numerical point of view. Indeed the numerical method needs to be able to capture complex pattern of shock waves and to accurately solve their interaction with turbulence. It is important that shock capturing algorithms used do not artificially damp turbulence.

In the present study, we apply a recently developed shock-capturing technique ${ }^{6}$ based on nonlinear artificial diffusivity to perform high-order simulations of jet penetration and mixing into a supersonic crossflows. ${ }^{7}$ The numerical method and the shock-capturing scheme are described in section II. Section III describes the flow configuration. In section IV preliminary results of the jet in supersonic crossflow configuration corresponding to a Mach 8 flight condition are presented and qualitative comparisons with experimental data are shown.

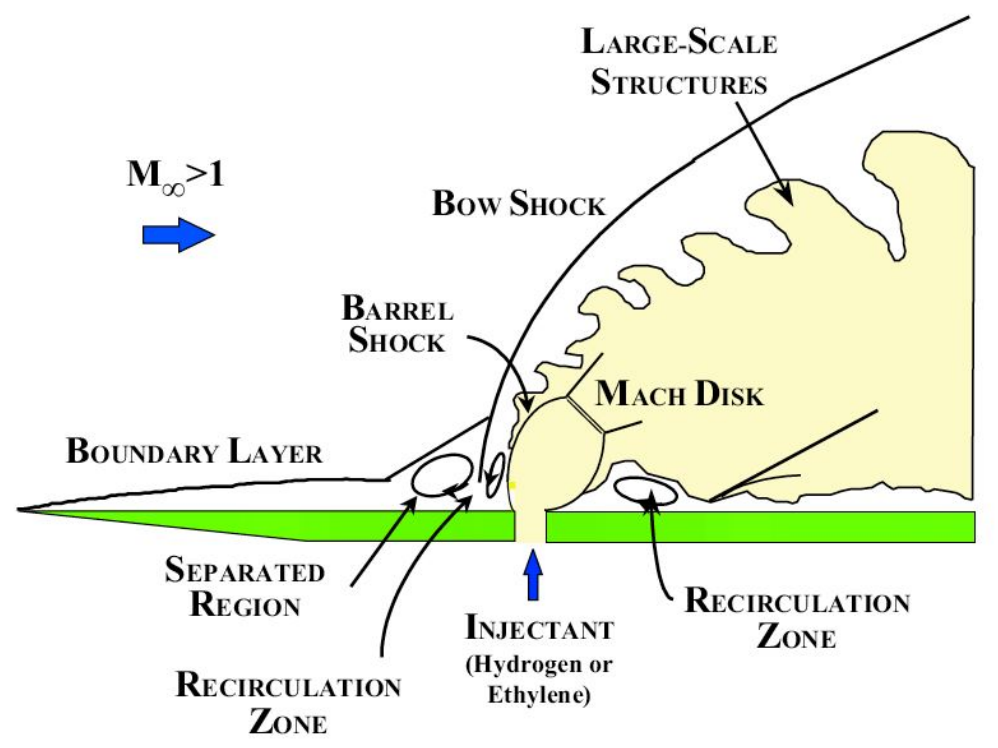

Figure 1. Schematic side view along the centerline of an under-expanded injection into a supersonic crossflow. ${ }^{7}$

\section{Numerical method: shock capturing scheme}

Due to their ability to accurately reproduce a wide range of wavenumbers, compact schemes ${ }^{8}$ are well suited for simulations of turbulent flows. Unfortunately, the use of high-order compact schemes to solve steep gradients like shock waves generates non-physical oscillations. ${ }^{9}$ To overcome this problem, several approaches which modify or adapt high-order schemes to treat flows with physical discontinuities have been 
proposed in the literature. ${ }^{10-14}$ An attractive strategy which is straightforward to implement in a CFD code and imposes low CPU time demand, consists of adding to the flow equations an artificial nonlinear diffusion term based on high-order derivative of the strain rate tensor. This method, originally proposed by Cook and Cabot, ${ }^{1,15}$ avoids the use of a shock detector and allows the same numerical scheme in smooth regions of the flow. This technique has been recently extended to supersonic reacting flows by Fiorina \& Lele. ${ }^{6}$ The key elements of this scheme are described below.

The equations of a non-reactive flow including the artificial diffusion terms are:

$$
\begin{array}{r}
\frac{\partial \rho}{\partial t}+\nabla \cdot \rho u-\nabla\left(\chi_{\rho} \nabla \rho\right)=0 \\
\frac{\partial \rho u}{\partial t}+\nabla \cdot(\rho \cdot u u+p \underline{\delta}-\underline{\tau})=0 \\
\frac{\partial \rho E}{\partial t}+\nabla \cdot[(\rho E u+(p \underline{\delta}-\underline{\tau}) \cdot u-\lambda \nabla T]=0 \\
\rho E=\frac{\rho R T}{\gamma-1}+\frac{1}{2} \rho u u,
\end{array}
$$

where $\underline{\delta}$ is the unit tensor, $\chi_{\rho}$ is a nonlinear artificial diffusivity and $\underline{\tau}$ is the viscous stress tensor given by:

$$
\underline{\tau}=\left(\mu_{s}+\mu_{l}\right)(2 \underline{S})+\left(\mu_{b}-\frac{2}{3}\left(\mu_{s}+\mu_{l}\right)\right)(\nabla \cdot u) \underline{\delta},
$$

where $\underline{S}=0.5\left(\nabla u+(\nabla u)^{T}\right)$ is the strain rate tensor, and $\mu_{l}$ is the fluid viscosity, $\mu_{s}$ and $\mu_{b}$ are respectively the grid dependent shear and bulk artificial nonlinear viscosity, defined as follow:

$$
\mu_{s}=C_{\mu}^{s} \eta, \quad \mu_{b}=C_{\mu}^{b} \eta, \quad \eta=\rho \Delta^{r+1} \overline{\left|\nabla^{r-1} S\right|},
$$

where $C_{\mu}^{s}$ and $C_{\mu}^{b}$ are the model constants, $\Delta$ is the local grid spacing and $S=(\underline{S}: \underline{S})^{1 / 2}$ is the magnitude of the strain rate tensor. $\nabla^{r-1}$ is the polyharmonic operator which denotes a sequence of Laplacians. For instance $r=5$ leads to $\nabla^{4} S=\nabla^{2}\left(\nabla^{2} S\right)$. The overbar $(\bar{f})$ denotes a truncated-Gaussian filter defined in Ref. 15. For practical meshes, the discrete representation of shock waves involves numerical discontinuities in the velocity and pressure field. In terms of Fourier analysis, these numerical discontinuities correspond to the largest wavenumbers. If $r$ is sufficiently high, $\mu_{s}$ and $\mu_{b}$ are expected to become important in the locations near shock wave and become close to zero in the rest of the flow. According to Cook and $\operatorname{Cabot}^{1}$, the splitting of the nonlinear viscosity into a shear and a bulk component allows capturing shocks without destroying vorticity. They also suggest that the non-linear viscosity model provides the correct rate of subgrid-scale energy transfer. Therefore no additional sub-grid model were used by Cook and Cabot for LES purposes.

The nonlinear artificial diffusivity $\chi_{\rho}$ introduced into Eq. 1 allows capture of strong contact surface discontinuities. ${ }^{6}$ We express $\chi_{\rho}$ in terms of high-order derivative of the fluid entropy $s$ :

$$
\chi_{\rho}=C_{\rho} \zeta, \quad \zeta=\frac{a_{0}}{c_{p}}(\Delta)^{r+1} \overline{\left|\nabla^{r-1}\right| \nabla s||},
$$

where $C_{\rho}$ is a model constant, $|\nabla s|$ is the norm of the fluid entropy gradient, $a_{0}$ is a reference speed of sound and $c_{p}$ is the specific heat at constant pressure.

Detailed analysis of the errors associated with shock-capturing and contact-surface capturing have been conducted for this scheme. ${ }^{6}$ These have shown that this approach is able to capture both weak and strong shocks without any degradation of performance. Both the number of points over which the discontinuity is smeared, and the damping of the spurious wiggles are largely independent of the mesh size and the shock/contact surface strength.

This numerical procedure has been implemented in the 3-D compressible Navier-Stokes solver FDL3DI ${ }^{16}$ developed at the Wright Patterson Air Force Research Laboratory. The spatial derivatives are computed with a 6th order compact scheme ${ }^{8}$ and a 8 th order filtering is used for stability purpose. ${ }^{16}$ The code is explicit in time using a fourth order Runge-Kutta method. Parameters used for the non linear viscosity model are $r=5, C_{\mu}^{s}=0.002, C_{\mu}^{b}=1$ and $C_{\rho}=0.01$. This set of model constants reduces the spurious wiggles amplitude without detrimental effect on the turbulence. 
A few test cases which demonstrates the performance of this scheme are discussed first. More complete discussion can be found in Ref.6. This shock capturing technique was applied to the reflection of a shock wave on an inviscid wall. The shock angle is 33 degrees from the Mach 3 free-stream. The mesh size is $151 \mathrm{x}$ 51 and is uniformly distributed in both directions. The jump conditions are imposed on the upper boundary whereas slip wall conditions are set at the bottom boundary. Pressure field is plotted in Fig 2(a) and 2(b). Although the shock wave is not aligned with the computational mesh, no significant wiggles are present around the discontinuity which is well captured. As a second example, a Mach 3 inviscid supersonic flow past a cylinder is computed. A $81 \times 75$ mesh, shown in Fig. 3(a) was generated analytically ${ }^{17}$ for the upper half of the domain. Symmetric flow conditions are imposed at the centerline. The problem is initalized by a Mach 3 shock moving from the left while slip wall conditions are imposed at the surface of the cylinder. Iso-contours of pressure are plotted in Fig. 3(a). Two radial profiles of pressure, corresponding to $\theta=0^{\circ}$ and $\theta=45^{\circ}$, where $\theta$ is defined in Fig. 3(a), are shown in Fig. 3(b). At the centerline, the shock, smeared over 4 grid points, is located at a distance of 1.7 from the cylinder, which is in good agreement whith the compact-Roe scheme results obtained by Visbal and Gaitonde ${ }^{14}$ on the same configuration. The maximal wiggles amplitudes are maintained below $2 \%$. The model has also been successfully applied to a double Mach reflection problem and a detonation wave propagation. The numerical setup and the results of these configurations are discussed in Ref. 6.

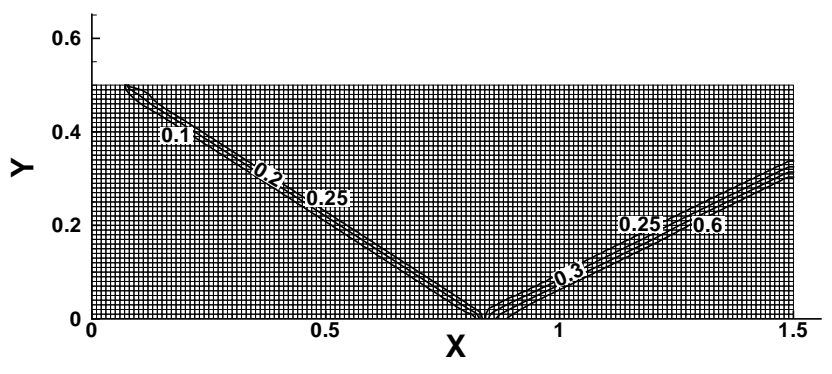

(a) Contours of normalized pressure

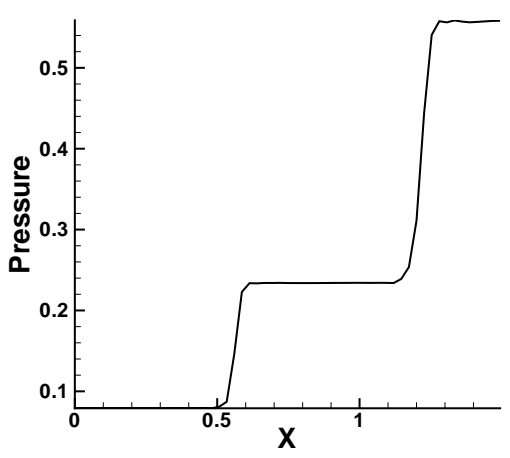

(b) Pressure along $\mathrm{Y}=0.18$.

Figure 2. The interaction of an oblique shock with an inviscid wall. The shock angle is 33 degrees from the Mach 3 free-stream.

\section{Jet in supersonic crossflow configuration}

Experiments of a hydrogen jet in a supersonic air crossflow have been performed in the Stanford expansion tube, in which a supersonic combustor environment for various flight Mach numbers (Mach 8-13) can be achieved. ${ }^{7,18,19}$ The free-stream flow conditions used in the experiments replicates SCRAMJET combustor entry conditions for flight Mach 10 . They are $T=1290 \mathrm{~K}, p=0.32 \mathrm{~atm}, \mathrm{~V}=2360 \mathrm{~ms}^{-1}$ and $M=3.4$. An under-expanded transverse jet of hydrogen with a $2 \mathrm{~mm}$ port diameter is injected into the Mach 3.4 free-stream flow at a distance $50 \mathrm{~mm}$ downstream a flat plat leading edge. Entry conditions of the jet are $T=240 K, p=0.49 M P, V=1205 \mathrm{~ms}^{-1}$ and $M=1.0$.

In this paper we present results from a preliminary simulation of this flow. To simplify the problem, the flow is assumed to be that of a single-component ideal gas. Both the free-stream and the jet are assumed to be uniquely composed of air. Earlier experimental studies showed that transverse penetration of the injected jet into the crossflow is mainly controlled by the jet-to-free stream momentum ratio. The same pressure, density and velocity conditions as in the experiment are set for both free-stream and jet, and in order to maintain the same jet-to-free stream momentum ratio, jet entry temperature is set to $3500 \mathrm{~K}$. The size of the computational domain is $10 \mathrm{D}$ X $10 \mathrm{D} \mathrm{X} 7.5 \mathrm{D}$ in the streamwise, spanwise and vertical directions, where $\mathrm{D}$ is the jet injector diameter. Corresponding number of grid points are $211 \mathrm{X} 101 \mathrm{X} 101$. The grid is 


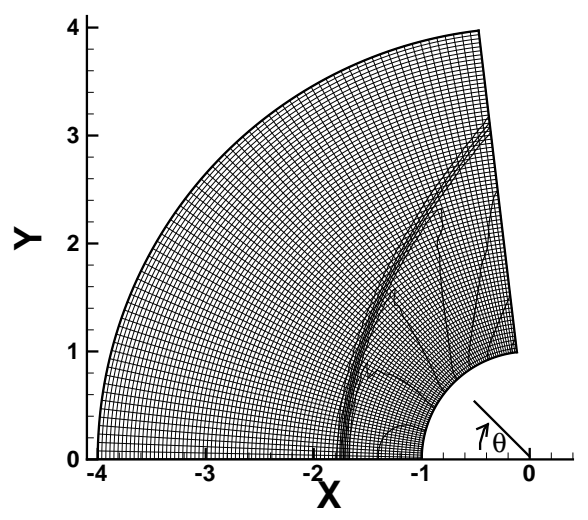

(a) Contours of normalized pressure

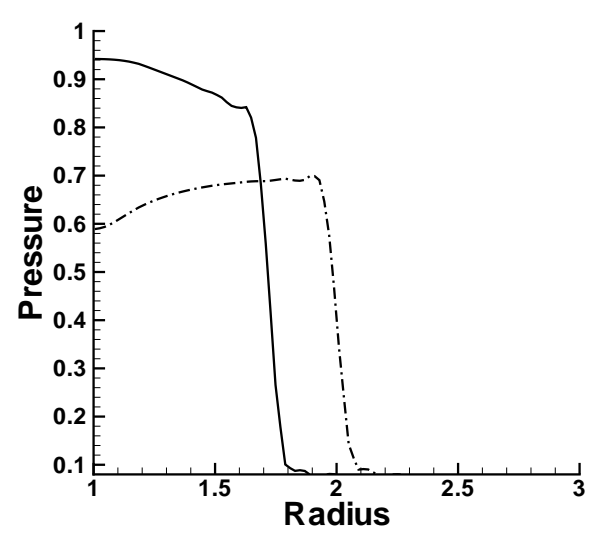

(b) Radial profiles of pressure. Solid line: $\theta=$ $0^{\circ}$; Dashed-dotted line: $\theta=45^{\circ}$

Figure 3. Mach 3 supersonic flow past a cylinder.

cartesian, clustered close to the wall and to the jet entry. Minimum and maximum grid spacing are $0.06 D$ (in $\mathrm{X}, \mathrm{Y}$ and $\mathrm{Z}$ directions) and $1.3 \mathrm{D}$ (in $\mathrm{Z}$ direction), respectively. Adiabatic no-slip boundary conditions are enforced at walls. For this preliminary study, the approaching boundary layer flow is assumed to be laminar. For the jet inlet boundary conditions, a turbulent mean profile is assumed. The axial velocity is given by $\langle\hat{u}\rangle=U_{c}(1-2 r)^{1 / n}$ where $n=7$ and $U_{c}$ is set to $1500 \mathrm{~ms}^{-1}$ in order to recover the correct jet mass flow rate.

The expressions of the non-linear viscosity and diffusivity by Eq. 6 and 7 are well suited for cartesian isotropic meshes. However, when the grid spacing is not identical for each spatial direction, the weighting of the high-order derivatives of the strain rate by a local grid spacing $\Delta$ doesn't guarantee the grid-dependent property of the non-linear viscosity. An alternative is to directly introduce the grid spacing in each direction in a slightly modified expression of the non-linear viscosity and diffusivity. For $r=5$, the modifed expression for $\eta$ and $\zeta$ become:

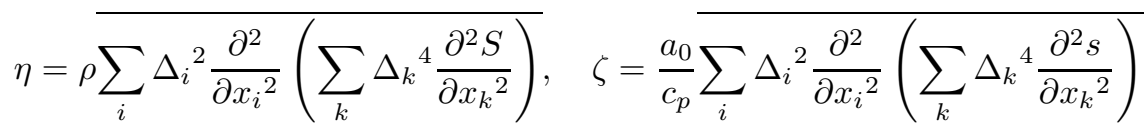

where $\Delta_{i}$ is the grid spacing in the $i$ direction.

\section{Results}

Schlieren images of this configuration (for two different injected gases) were reported in the experiments conducted by Ben Yakar et al. ${ }^{19}$ The long time averaged flow fields properties are shown in Fig. 4(a) where the flow was imaged with a long exposure time of $3 \mu \mathrm{s}$. Note the convective time scale $t_{j}=D_{j} / V_{j}$ is 0.85 $\mu s$ and the boundary layer convective time scale $t_{\delta}=\delta / V_{\infty}=0.2 \mu s$. The plot of the norm of mean density gradient in Fig. 4(b) allows to qualitatively compare the general flow features with the experimental schlieren picture shown in Fig. 4(a). Clearly visible in both numerical simulation and schlieren image is the oblique bow shock, the expanding core of the injected jet and the separation shock wave. The contours of the magnitude of the mean velocity and streamlines along the centerline predicted by the LES are shown on Fig. 5(a). These data are obtained by averaging over $6.4 \mu \mathrm{s}$ of the flow calculation. This averaging time is short and should be kept in mind in interpreting the results to be discussed. The barrel shock and the Mach disk, not very clear in the long exposure schlieren image, are however highlighted by the numerical simulation. Streamlines based on the mean velocity shows three recirculation zones upstream of the injection hole. The one closest to the jet injection, the so-called horseshoe vortex (HSV), has an omega-shape structure that 
wraps the jet column and stays fairly close to the plate surface. This vortex is also visible on Fig.5(c) and $5(\mathrm{~d})$, where the contours of the magnitude of the mean velocity and streamlines at two streamvise locations are shown. Initially formed on each lateral edge of the jet, the counter-rotating vortex pair (CWP) is also identified on these figures.

Figure 6(a) shows a comparison between measurement and prediction of the mean bow shock position. Good agreement between experiment and simulation is observed in the region away from the wall and demonstrates the performance of the shock-capturing scheme. However some discrepancy is observed in the near wall region of the bow shock that may be explained by the following reasons. The flow in the near wall region upstream to the injection hole is unsteady and insufficient statistics may explain some of the discrepancy. This unsteadiness in the bow shock position is illustrated by the horizontal red line located at $Y / D=0.7$ on Fig. 6(a) which shows the range in which the bow shock evolves. Further more the present calculations use a laminar boundary profile upstream of the jet injection, whereas the actual flow is most likely to be turbulent and this may significantly influence the shock-boundary layer interaction. Also to note is that the present calculations specify conditions at the exit of the nozzle, i.e. they do not model the internal flow within the nozzle. This may also influence some detail of the interaction.

Positional fluctuations and curvature changes in the bow shock has been previously observed by Gruber and Nejad. ${ }^{20}$ Changes in the position of the bow shock are visualized on Fig. 6(b) where pressure profiles across the bow shock along the centerline at a constant $Z / D=0.7$ location are plotted. A sequence of time steps which describes one period of bow shock motion is shown. Starting from the most upstream position limit (taken as time $t=0 \mu \mathrm{s}$ ), the shock moves downstream, and reaches its closest location to the jet injection at half a period $(t=3.2 \mu s)$. During the second half period, the shock goes back to its initial state. Experimental investigations of this flow configuration showed that the bow shock behavior below the point of intersection with the separation shock is strongly influenced by the structures formed after the jet enters the crossflow. ${ }^{20}$ These are large scale shear layer vortices formed at the jet/freestream interface ${ }^{19-21}$ that play an important role in fluid macro-mixing. In Fig 7 snapshots of the entropy contours in the centerline plane are presented. At $\mathrm{t}=0$, no eddies are observed and the shock stands at it most upstream location. At $t=1.6 \mu \mathrm{s}$ , an eddy (1) is formed that increase the curvature of the bow shock which causes it to move towards the jet exit. At $t=3.2 \mu s$, eddy (1) has been convected downstream and a second eddy (2) is formed that still affects the bow shock curvature. The angle between the shock and the freestream increase that cause an increase in pressure ratio across the shock, as shown on Fig. 6(b). At $t=4.8 \mu \mathrm{s}$ the second eddy is released. As no additional eddies are generated, the shock is able to recover to its original state at $t=6.4 \mu s$. The shock angle decrease that cause the pressure ratio across the shock to decrease as well. Both experimental ${ }^{19}$ and the present numerical investigation show that the formation of the eddies is not at a fixed frequency. A rigorous validation of the frequency of the eddies formation is therefore not straightforward, however the same order of magnitude as seen in the experiments is recovered. This qualitative flow description of the preliminary results presented here illustrates the nature of the bow shock / jet interactions. Higher resolution calculations with longer simulation time are planned for the future that will allow a more quantitative analysis of the shear shear layer vortex formation and convection. The need for simulation with a mesh better adapted to resolve the shear layer vortices can be seen from figure 7 (f) which overlaps every $4^{\text {th }}$ grid point on the center plane entropy contours. It is immediately evident that the grid resolution at both edge of the jet is quite marginal. Despite this the present calculation provides some useful qualitative insights.

Finally a three-dimensional visualization of the complex flow patterns is presented in Fig.8. Iso-contours of entropy and streamlines of the vorticity vector are shown that indicate the orientation of the vortex core. The blue lines represent the horseshoe vortex that is formed upstream of the jet column further wraps around injected flow in the boundary layer. The purple streamlines represent the secondary horseshoe vortex that is formed further upstream. Red colored streamlines highlight the large scale coherent structures that are located at the jet-free stream shear layer interface. Although the large scale eddies seem to be two-dimensional in Fig. 7, they are part of the unsteady Kelvin-Helmholtz circumferential rollers wrapping around the jet. They have a spanwise rolling shape that surround the jet and also the horseshoe vortices. Finally the counter rotating vortex pair is identified by the black line.

\section{Conclusion}

A shock-capturing approach based on localized artificial dissipation has been used to perform computation of a jet penetration into a supersonic crossflow with a high-order accurate finite difference scheme. 


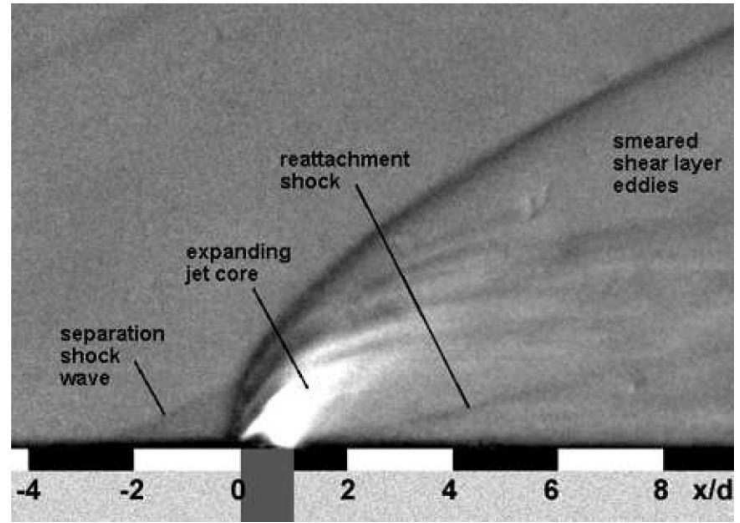

(a)

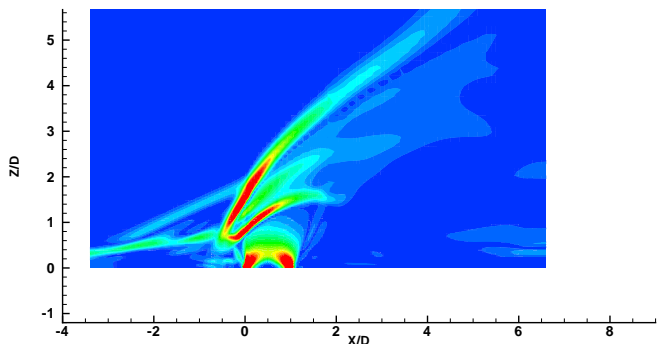

(b) Norm of mean density gradient

Figure 4. Experimental $^{7}$ (left) and numerical (right) schlieren picture of the jet in supersonic crossflow configuration along the centerline plane.

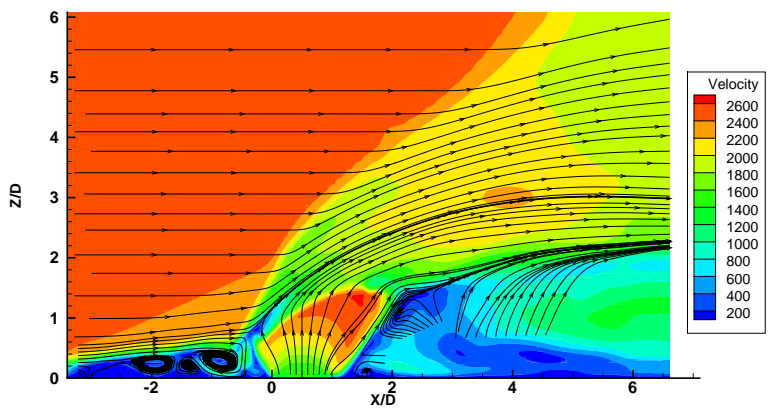

(a) Centerline plane

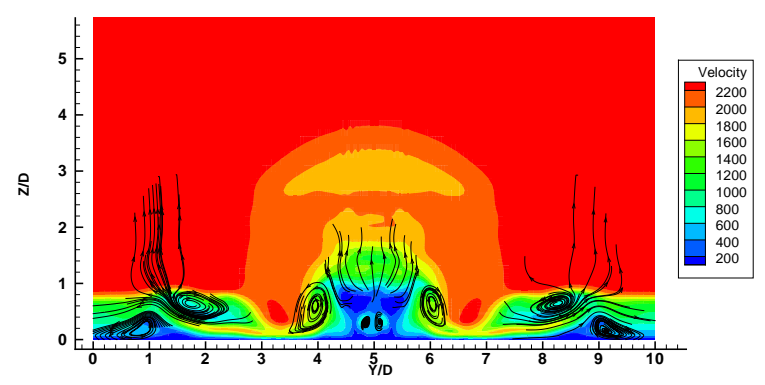

(c) $Y / D=2$ plane

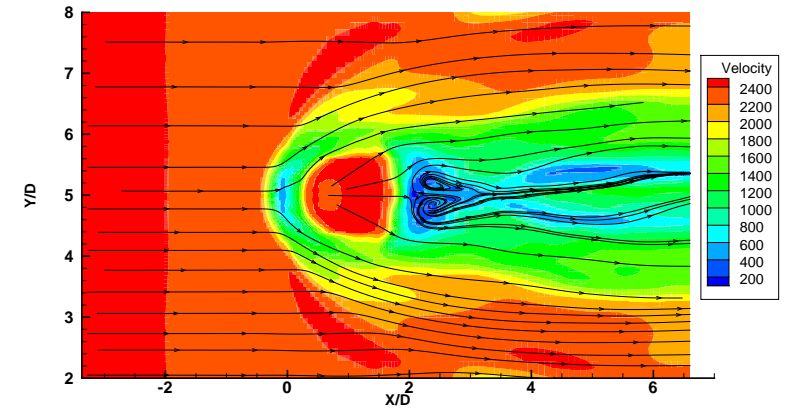

(b) $Z / D=1$ plane

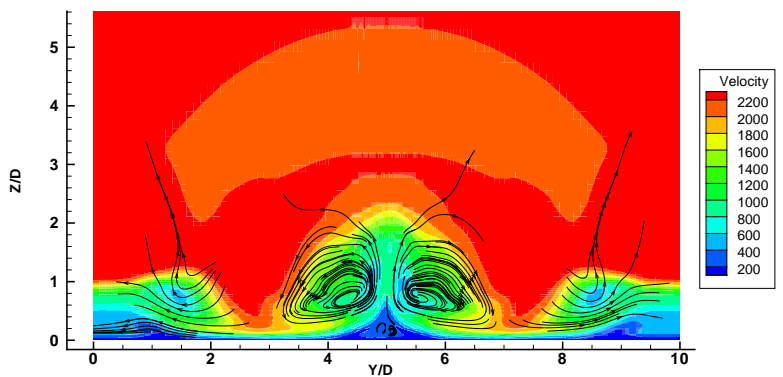

(d) $Y / D=4$ plane

Figure 5. Contours of the magnitude of the mean velocity (in $\mathrm{ms}^{-1}$ ) and streamlines for the jet in crossflow simulation 


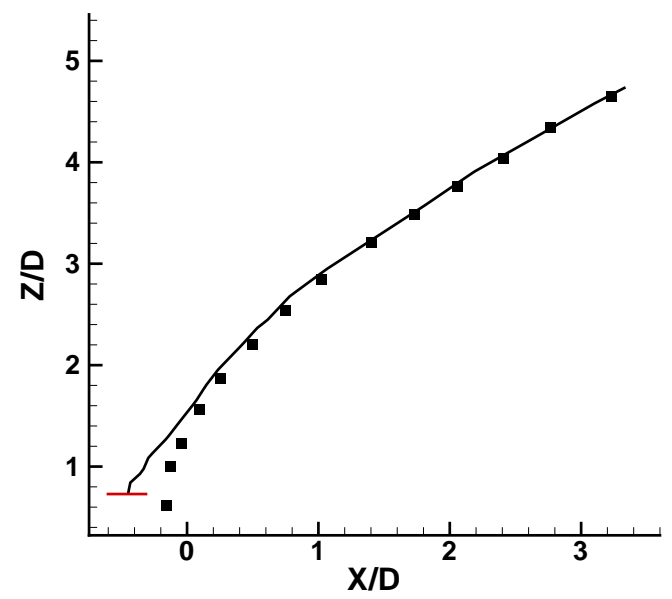

(a) Mean bow shock position. Symbols: experimental data. Line: LES
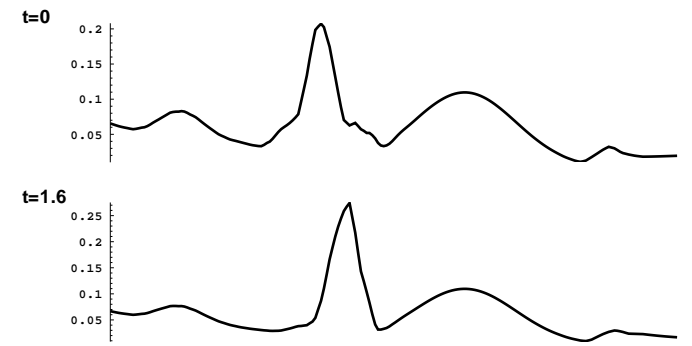

$\mathrm{t}=3.2$

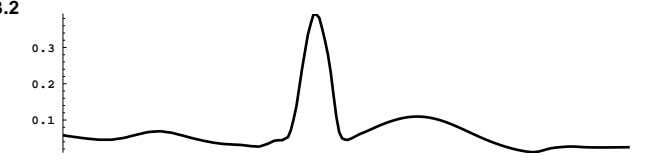

$\mathrm{t}=4.8$

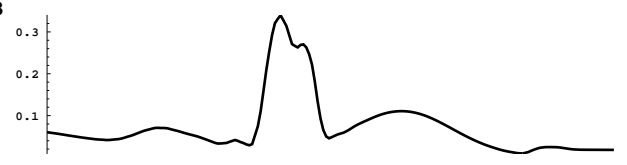

$t=6.4$

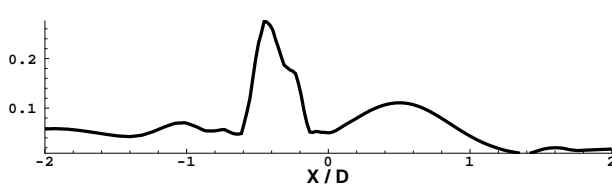

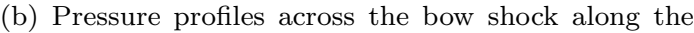
centerline at a constant $z / D=0.7$

Figure 6.

8 of 11 


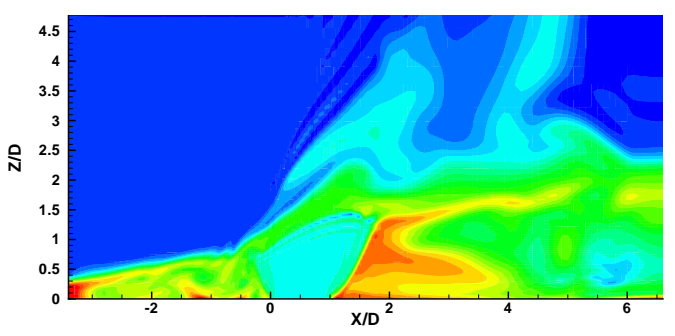

(a) $t=0 \mu \mathrm{s}$

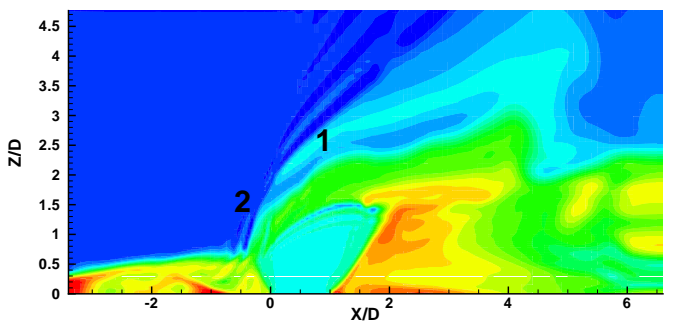

(c) $t=3.2 \mu \mathrm{s}$

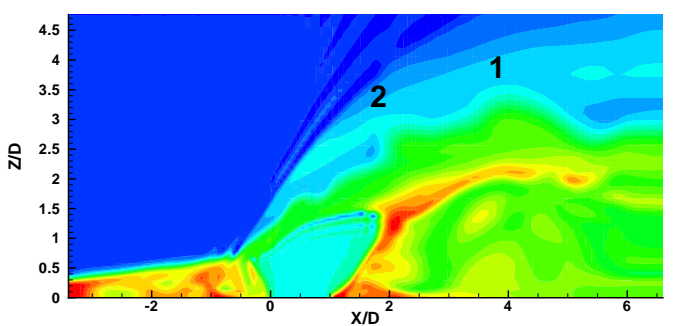

(e) $t=6.4 \mu \mathrm{s}$

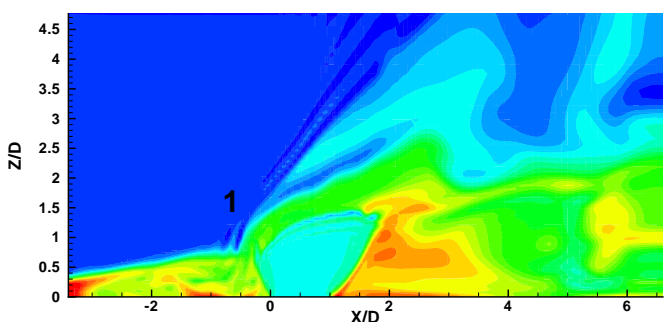

(b) $t=1.6 \mu \mathrm{s}$

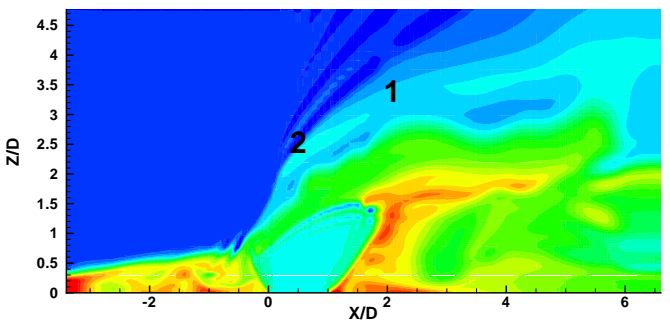

(d) $t=4.8 \mu \mathrm{s}$

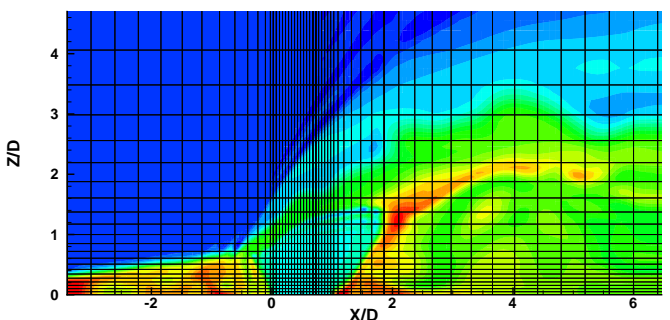

(f) $t=6.4 \mu \mathrm{s}$

Figure 7. Instantaneous entropy contours along the centerline plane. The mesh is shown for every fourth grid point on (f). 


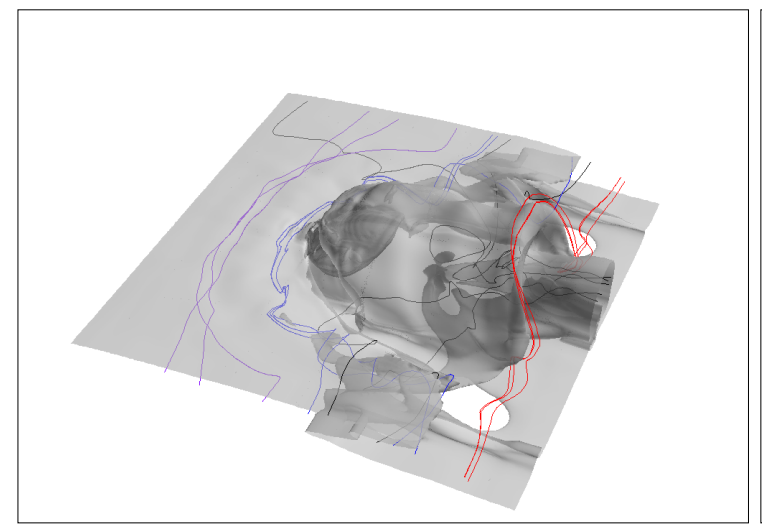

(a)

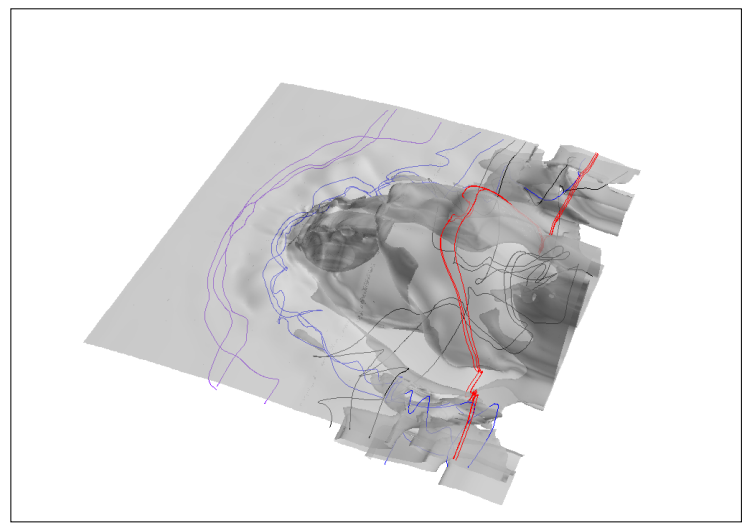

(b)

Figure 8. 3D views of entropy iso-contour and vorticity streamlines. Blue and purple lines are horseshoe vortices, red lines are the spanwise rollers and black lines are the counter-rotating vortex pair.

Preliminary results have shown that main features of the flow are recovered. In our current work, we focus on including turbulence effects by performing large eddy simulations of this configuration. In future, the computational method will be extended to include appropriate models of combustion in the LES framework.

\section{Acknowledgments}

This work was supported by the AFOSR-MURI grant. We are grateful to Dr. Miguel Visbal for providing the FDL3DI code which was modified in the present work. We also acknowledge the efforts of Dr. Daniel Bodony in porting this code.

\section{References}

${ }^{1}$ Cook, A. W. and Cabot, W. H., "Hyperviscosity for shock-turbulence interactions," J. Comput. Phys., Vol. 203, No. 2, 2005, pp. 379-385.

${ }^{2}$ Andreadis, D., Drake, A., Garrett, J. L., Gettinger, C. D., and Hoxie, S., "Design considerations of ISTAR hydrocarbon fueled combustor operating in air augmented rocket, ramjet and scramjet modes." 11th AIAA/AAF International Conference on Space Planes and Hypersonic Systems and Technologies, Orleans, France, September 29, 2002.

${ }^{3}$ Mitani, T. and Kouchi, T., "Flame structures and combustion efficiency computed for a Mach 6 scramjet engine," Combust. Flame, Vol. 142, No. 3, 2005, pp. 187-196.

${ }^{4}$ von Lavante, E., Zeitz, D., and Kallenberg, M., "Numerical simulation of supersonic airflow with transverse hydrogen injection," J. Propulsion Power, Vol. 17, No. 6, 2001, pp. 1319-1326.

${ }^{5}$ Peterson, D. M., Subbareddy, P. K., and Candler, G. V., "DES Investigations of Transverse Injection into Supersonic Crossflow using a Hybrid Unstructured Solver," AIAA paper, 44th AIAA Aerospace Sciences Meeting and Exhibit., Vol. 2005$1265,2006$.

${ }^{6}$ Fiorina, B. and Lele, S. K., "An artificial nonlinear diffusivity method for supersonic reacting flows with shocks," Submitted for publication, 2005

${ }^{7}$ Ben-Yakar, A., Experimental investigation of mixing and ignition of transverse jets in supersonic crossflows, Ph.D. thesis, Department of Mechanical Engineering, Stanford University., 2000.

${ }^{8}$ Lele, S. K., "Compact finite difference schemes with spectral-like resolution," J. Comput. Phys., Vol. 103, 1992 , pp. 16-42.

${ }^{9}$ Colonius, T. and Lele, S. K., "Computational aeroacoustics: progress on nonlinear problems of sound generation," Progress in Aerospace Sciences, Vol. 40, 2004, pp. 345-416.

${ }^{10}$ Adams, N. A. and Shariff, K., "Direct numerical simulation of the turbulent boundary layer along a compression ramp at $\mathrm{M}=3$ and $\mathrm{Re}=1685$," J. Fluid Mech., Vol. 127, 1996, pp. 27-51.

${ }^{11}$ Pirozzoli, S., "Conservative Hybrid compact-WENO schemes for shock-turbulence interaction." J. Comput. Phys., Vol. 178, 2002, pp. 81-117.

${ }^{12}$ Deng, X. G. and Zhang, H. X., "Developing high-order weighted compact nonlinear schemes," J. Comput. Phys., Vol. 165, 2000, pp. 22-44.

${ }^{13}$ Rizzetta, D. P., Visbal, M. R., and Gaitonde, D. V., "Large-eddy simulation of supersonic compression ramp flow by high-order method," AIAA Journal, Vol. 39, No. 12, 2001, pp. 2283-2292. 
${ }^{14}$ Visbal, M. R. and Gaitonde, D. V., "Shock capturing using compact-differentiated-bases method," AIAA paper, 43rd AIAA Aerospace Sciences Meeting and Exhibit., Vol. 2005-1265, 2005.

${ }^{15}$ Cook, A. W. and Cabot, W. H., "A high-wavenumber viscosity for high-resolution numerical methods," J. Comput. Phys., Vol. 195, No. 2, 2004, pp. 594-601.

${ }^{16}$ Gaitonde, D. V. and Visbal, M. R., "High-order schemes for Navier-Stokes equations: algorithm and implementation into FDL3DI. Technical Report AFRL-VA-WP-TR-1998-3060," Tech. rep., Air Force Research Laboratory, Wright-Patterson AFB, 1998.

${ }^{17}$ Jiang, G. S. and Shu, C. W., "Efficient implementation of weighted ENO scheme." J. Comput. Phys., Vol. 126, 1996, pp. 202-228.

${ }^{18}$ Ben-Yakar, A. and Hanson, R. K., "Experimental investigation of flame-holding capability of a transverse hydrogen jet in supersonic crossflows," The Proceedings of the 27th Symposium (Int.) on combustion, Vol. 40, 1998, pp. 435-416.

${ }^{19}$ Ben-Yakar, A., Mungal, M. G., and Hanson, R. K., "Time evolution and mixing characteristics of hydrogen and ethylene transverse jets in supersonic crossflows," Physics of fluids, Vol. 18, 2006.

${ }^{20}$ Gruber, M. R. and Nejad, A. S., "Bow Shock/Jet Interaction in Compressible Transverse Injection Flowfields," AIAA journal, Vol. 34 (10), 1996, pp. 2191-2193.

${ }^{21}$ Gruber, M. R., Nejad, A. S., Chen, T. H., and Dutton, J. C., "Large structure convection velocity measurements in compressible transverse injection flowfields," Experiments in Fluids, Vol. 22, 1997, pp. 397-407.

11 of 11 